\title{
Role of Essential Fatty Acids in Human Nutrition
}

\author{
Ralph T. Holman \\ The Hormel Institute, University of Minnesota \\ (Austin, Minnesota 55912, U.S.A.) \\ Presented before the Japan Oil Chemists' Society, \\ November 1, 1974, at Osaka University
}

In 1929 Professor George Burr and his wife $\mathrm{e}^{1)}$ published the first report that fat was a necessary component of the diet of rats. They showed that a low-fat diet caused poor growth, scaly skin and tail, and early death, and also that linoleic acid prevented or cured these conditions. Immediately thereafter students and associates of Professor Burr attempted to study the role of polyunsaturated acids (PUFA) in the diet of humans. Dr. W.R. Brown ${ }^{2)}$ was fed a low-fat diet for several months, and the iodine value of his serum lipids decreased slightly and the only clinical effect he noticed was that his migraine headaches disappeared! We now know that the diet he ate was not really free of essential fatty acids (EFA), and that a much longer time would be required to induce EFA deficiency in adults. This experiment created the impression that EFA were of minor importance in adult human nutrition, and this opinion persisted in the medical community until about a decade ago.

The most obvious symptom of EFA deficiency in animals is a scaly skin, and it was natural to seek a connection between dermatitis and EFA deficiency in humans. Dr. Arild Hansen ${ }^{3)}$, a student of Burr and a pediatrician, showed in the 1930 's that many cases of infant eczema which did not respond to the usual treatment for eczema, did respond to a dietary supplement of lard. He observed that such cases had a lower total unsaturation of the serum lipids than normal, and that when they were cured by dietary lard, the unsaturation of serum lipids was restored to normal. One such case is shown in Fig.-1, before and after a dietary supplement of lard. The microscopic changes in the skin are shown in Fig.-2. A is the skin of a normal infant and $B$ the skin of an infant fed a milk formula containing less than 0.1 $\%$ of the calories as linoleic acid ${ }^{4}$. Hansen and his coworkers continued these studies and showed that

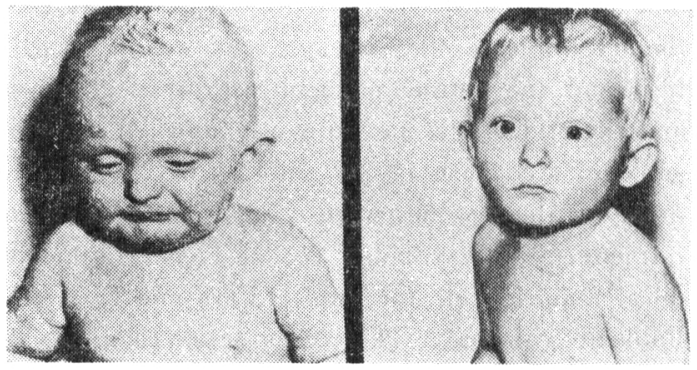

Fig.-1 Child with intractable eczema before and after dietary treatment with supplements of lard.

(B)

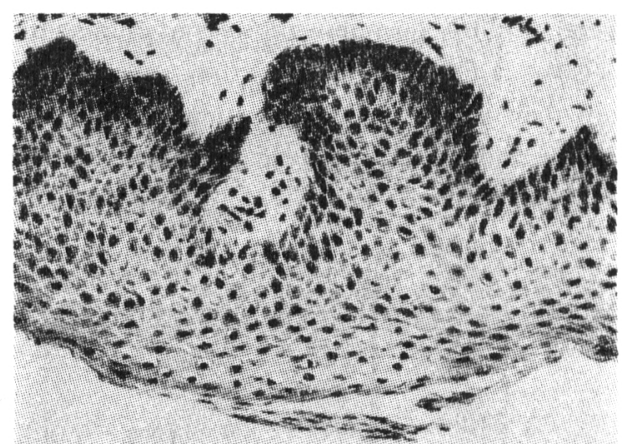

(A)

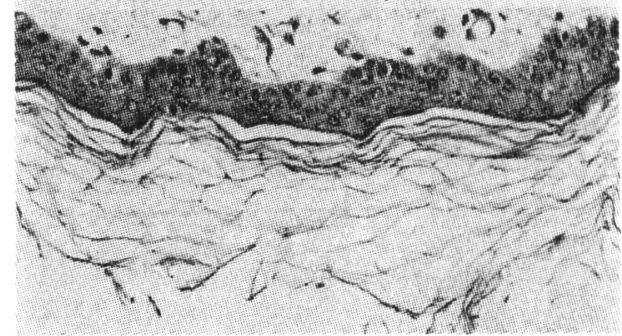

Fig.-2 Histological sections of skin from EFA-defiicient infant (above) and normal infant (below). 
in cases of infant eczema which responded to dietary EFA, the dienoic and tetraenoic acids of serum lipids were lower, and trienoic acids were higher than in normal infants, using alkaline isomerization as the method of analysis.

In 1959 our laboratory began a series of studies in which the effects of dose level of EFA were studied, rather than simple comparison of deficient and supplemented animals. The first of these studies involved alkaline isomerization as the analytical method, and this method was later compared with gas chromatography. The results of the two methods were fortunately found to be quite the same. In the first of these studies, the proportions of trienoic acid (5,8,11-eicosatrienoic acid) and tetraenoic acid (arachidonic acid, 5,8,11,14eicosatrienoic acid) in heart and liver were measured as a function of dietary intake of linoleic acid. The results showed that below $0.5 \%$ of calories of dietary linoleate, the trienoic acid was abnormally high and the tetraenoic acid was unusually low ${ }^{5}$ and the triene/tetraene ratio was above 1 . In rats supplemented with $1 \%$ of calories of linoleic acid or more, these values were normal and the triene/ tetraene ratio was below 0.4 . Thus, the biochemical requirements for linoleate are met by $1 \%$ of linoleate calories. This was later found to be true for many tissues of rats, and for many species of animals. This is shown in Fig. $-\mathbf{3}$.

Hansen and his group attempted to evaluate several infant formula foods containing different levels of EFA. In their study, which involved more than 400 infants, they found that skim milk formula induces EFA deficiency, judged by skin condition and by the content of trienoic and tet-

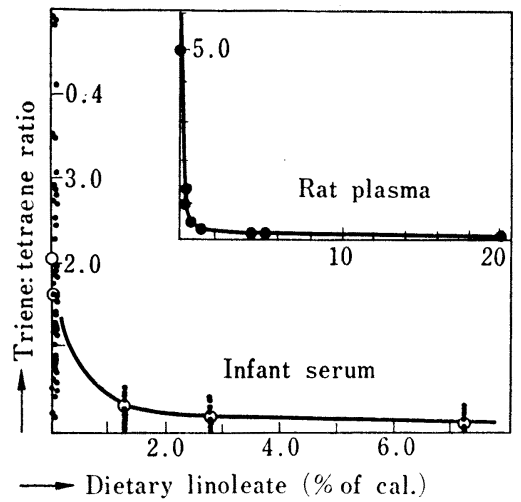

Fig.-3 Triene/Tetraene ratio in total lipids of rat plasma and of infant serum plotted against intake of linoleate. raenoic acids in serum lipids ${ }^{6}$. When the data of Hansen and his co-workers for infants was treated in the same manner as we treated the data from rats, the same relationship was found ${ }^{7)}$. In Fig.3 the triene/tetraene ratios in rat plasma and in infant serum are plotted against linoleate intake, and the two curves are nearly identical. The abrupt change in triene/tetraene ratio occurs near $1 \%$ of calories, and this is now considered the minimum requirement for linoleic acids in both animals and man.

The same data from Hansen's infants has been used to develop an equation by which it is possible to calculate how well an individual meets the requirement for $\mathrm{EFA}^{7)}$. The relationship between $\log$ dose of linoleate and the content of dienoic, trienoic and tetraenoic acids in serum lipids is shown in Fig.-4. These data and this equation permit estimate of linoleate intake to within about $25 \%$ of the true value. This is precise enough for the practical purpose of deciding whether an infant is deficient (less than $1 \%$ of calories) or is in the normal range $>5 \%$ of calories).

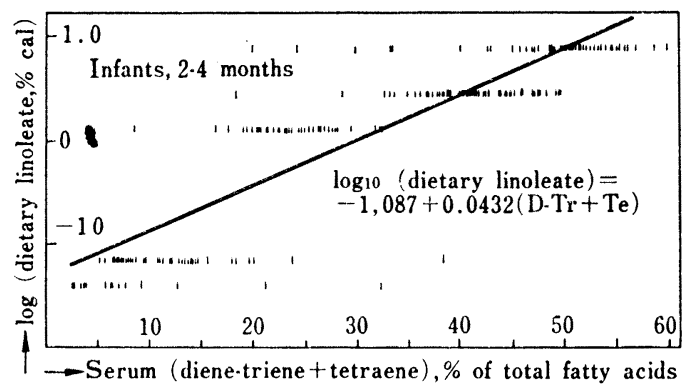

Fig.-4 The relationship between dietary linoleate and the algebraic sum of dienoic acidstrienoic acids + tetraenoic acids in serum of infants.

A similar study was made of 12 young adult males $^{8}$, and the estimation equation for them is shown in Fig.-5. In this study it was not possible to feed really deficient diets, so we were unable to set the EFA requirement for adult males. We believe it is probably very close to the requirement for children. Judging from our studies on rats, the EFA requirement for females may be less than the requirement for males ${ }^{9}$.

If the apparent dietary linoleate, calculated as [diene-triene + tetraene] of serum, is plotted against the age of breast-fed infants, newborn infants 


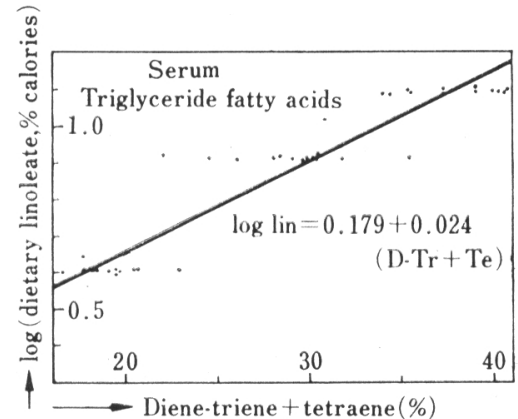

Fig.-5 Relationship between dietary linoleate and the sum of diene-triene-tetraene acids in serum of young adult males.

appear to be EFA deficient, and their serum approaches the normal values for PUFA within a few days $^{10)}$. This is shown in Fig.-6. However, if young infants are fed only cow's milk formula, the triene/tetraene ratio increases, whereas in breastfed infants the ratio remains low, suggesting that cow's milk may induce low level EFA deficiency in infant $\mathrm{s}^{\left.1^{0}\right)}$.

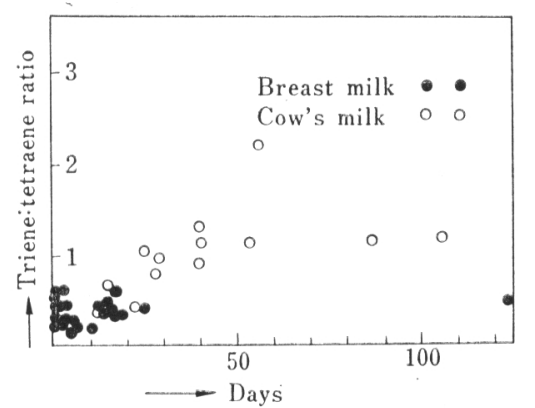

Fig.-6 The effects of breast feeding and cow's milk formula upon triene/tetraene ratio in infant serum lipids.

There is some evidence that faulty metabolism of EFA may be a factor in some rare diseases. Achrodermatitis enteropathica is an infant disease involving severe dermatitis and malabsorption. In his lengthy study of this disease, Dr. Ralph Cash of Detroit has concluded that the cause is an error in absorption or metabolism of linoleic acid, and he uses intravenous infusion of fat emulsion as a diagnostic tool ${ }^{11)}$. In Fig. -7 one of his cases is shown at the time of admission to the hospital (A). After a few days of intravenous fat emulsion, the dermatitis had improved significantly (B). Withdrawal of the fat emulsion for a few days caused the return of the dermatitis (C). The diagnosis was thus made that the case was Achro- dermatitis enteropathica, which usually responds to a diet containing no cow's milk and to daily administration of Diodoquin. After several days on this therapeutic regime, the dermatitis disappeared (D).

The fatty acid composition of the serum lipids was analyzed by GLC at each stage of the diagnosis and treatment, and the analyses confirmed in our laboratory. In conditions $\mathrm{A}$ and $\mathrm{C}$, the lipids had a low content of arachidonic acid and a high content of unusual fatty acids, perhaps odd chain and branched chain fatty acids. When the condition was improved by intravenous fat emulsion (B) or by Diodoquin and no cow's milk (D), the arachidonic acid increased toward normal level and the unusual fatty acids decreased. The case was clearly one of abnormal lipid absorption or metabolism.

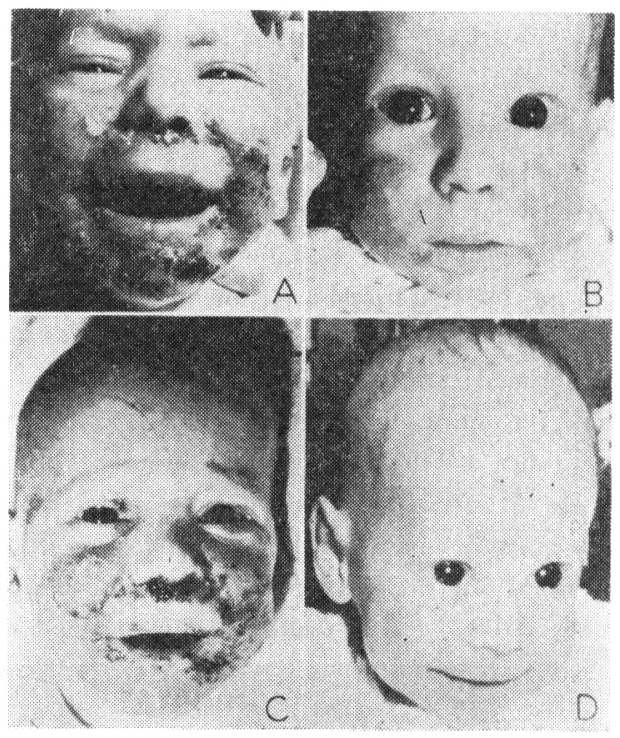

Fig.-7 A case of Achrodermatitis enteropathica.

The association with Dr. Cash led to acquaintance with Dr. Whitten, also of Detroit, who was caring for a case of radical bowel resection and who suspected the child was developing EFA deficiency. The child had experienced extreme intestinal upset shortly after birth, and exploratory surgery discovered that the mesentery had rotated at birth, cutting off the blood supply and killing the intestine. Joining of the duodenum to the colon left the child with very little absorptive intestine and constant intravenous feeding was required to support life. The usual intravenous infusion consists of glucose, amino acids, minerals and vita- 
mins, but no fat. It is therefore an extremely EFA deficient diet. When I saw the child at 3 months of age, it had already the extensive dermatitis, shown in Fig.-8. Samples of serum taken serially were analyzed for fatty acid composition of the triglycerides, cholesteryl esters and phospholipids, and very abnormal PUFA patterns and severe EFA deficiency were found. The triene/tetraene ratio at about 100 days of age was 16to be compared with 6 for the most severely deficient rat we had ever analyzed ${ }^{12}$.

This observation led to a study of 7 infants which, for one reason or another, required long-term intravenous feeding. They uniformly underwent the same changes in PUFA pattern. The changes in PUFA content

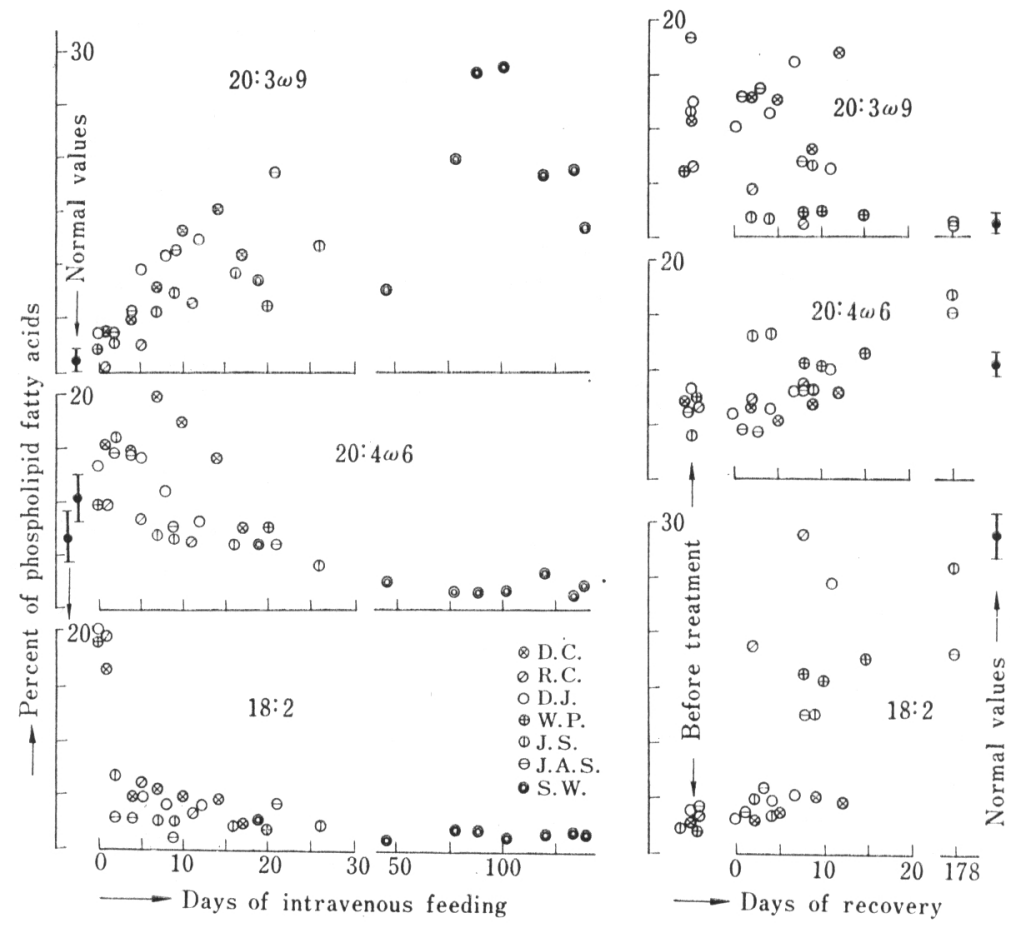

Fig.-9 Changes in PUFA content of infant serum phospholipids induced by long term intravenous feeding with a fat-free preparation, and recovery after feeding normal infant food.

of serum phospholipids are shown for the 7 infants

in Fig.-9. The upward trend for $20: 3 \omega 9$ (eicosatrienoic acid) and the downward trend for $20: 4$ (1) 6 is the same for each individual, although the magnitude of the change is different for each. For those fortunate children who could later be given normal food, the abnormal patterns changed to normal, indicating that these biochemical changes are reversible. The severe case mentioned above

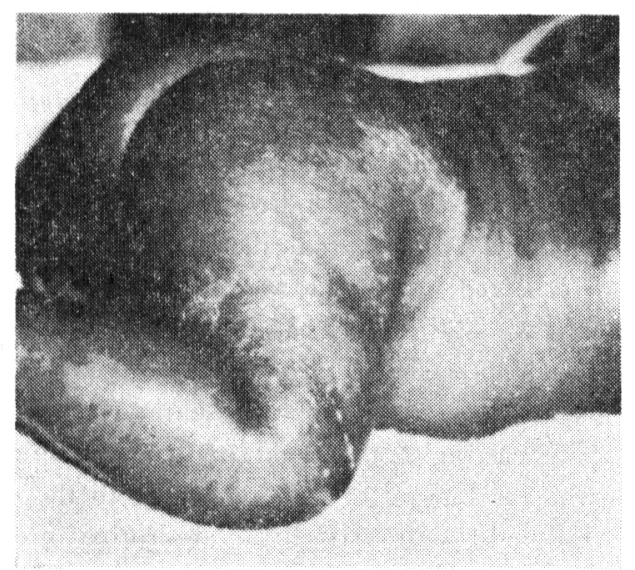

Fig.-8 Dermatitis of EFA deficiency induced in an infant by long term intravenous alimentation with a fat-free preparation. is shown in the chart with double circles (S.W.).

This child lived for 4.5 months, and was fed continuously by intravenous infusion. At autopsy, samples of tissue were taken, the fatty acid compositions of tissue lipids were analyzed, and the results showed that in all tissues the fatty acid characteristic of EFA deficiency, 5,8,11-eicosatrienoic acid, is present in very high proportions, and arachidonic acid is present in abnormally low amounts. This case is the most completely des. criber, and most severe case of EFA deficiency in a human thus far reported ${ }^{12)}$. The similarity in fatty acid composition to purposely induced EFA deficiency in rats striking, and is proof that EFA deficiency can be induced in man.

A report of a similar case in a 44 year old man has been made from Australia by Collins and his colleagues ${ }^{13)}$. They followed the content of $20: 3$ and $20: 4$ in serum lipids during two cycles of fat-free intravenous feeding followed by administration of a fat emulsion. Their results are shown in Fig. -10 . When fat-free intravenous fluid was given, 20:3 was high and 20:4 was low. When fat emulsion containing linoleate was given, $20: 3$ dropped to normal and $20: 4$ increased 


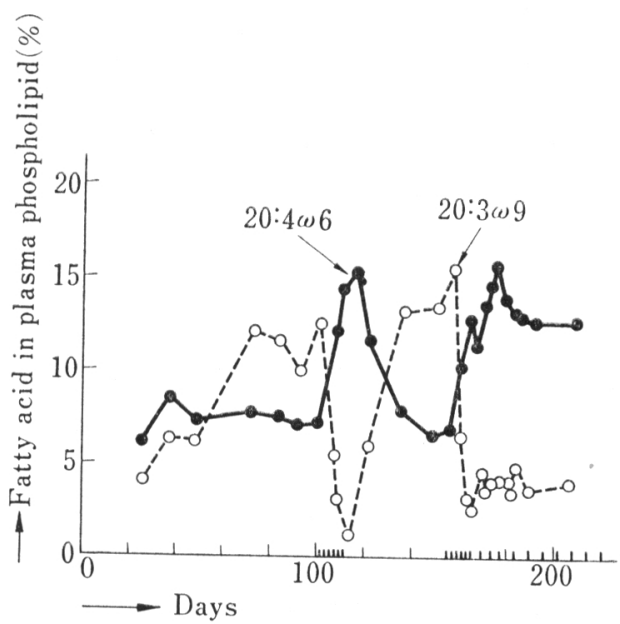

Fig.-10 The effects of intravenous feeding without and with fat emulsion in a 44 year old man. The administration of fat emulsion is indicated by marks on the time axis.

to normal. It is clear that intravenous fat emulsion prevents the biochemical deficiency of EFA.

EFA has value in human physiology beyond prevention of dermatitis. EFA is required in large amounts during periods of growth of tissue. Hence, EFA deficiency can be induced in infants and young animals much more easily than in adults. Other conditions involving proliferation of tissue might be thus expected to require EFA in increased amount. Indeed, Caldwell and his co-workers found wound healing to be defective in infants who have been maintained for long periods of time on fat-free intravenous nutrient ${ }^{14)}$. Fig. -11 shows the surgical wounds of a child which underwent repeated surgery for bowel resection and whose wounds did not heal during the period it was fed intravenously a fat-free preparation, and the same child after it had been fed for some

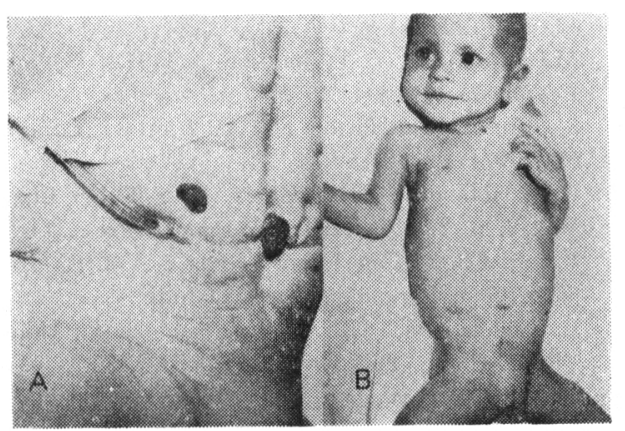

Fig.-11 Surgical wounds of an infant fed intravenously with a fat-free preparation, and after administration of an emulsion of fat. weeks with fat emulsion containing EFA. Thus, EFA is required for proper wound healing. There is also reason to suspect that EFA deficiency makes bacterial invasion easier, and that this tendency toward infection is increased in long-term intravenous feeding with fat-free preparations.

Recently a group of British workers has demonstrated that EFA deficiency which had been induced by long-term intravenous feeding with a fat-free preparation, could be relieved by absorption of unsaturated oils through the skin. Press and his co-workers showed that rubbing sunflower seed oil into the skin caused sufficient absorption to not only relieve the dermatitis of deficiency, but to reverse the abnormal pattern of PUFA in serum lipids ${ }^{15)}$. Fig. -12 shows that within a few weeks, the daily application of oil to the skin caused a significant reduction of $20: 3 \omega 9$ and an increase in $20: 4 \omega 6$ in serum lecithin. Thus, the oil was absorbed in sufficient amount to cure the biochemical deficiency. It may not then be necessary to administer unsaturated fat by the hazardous intravenous route.

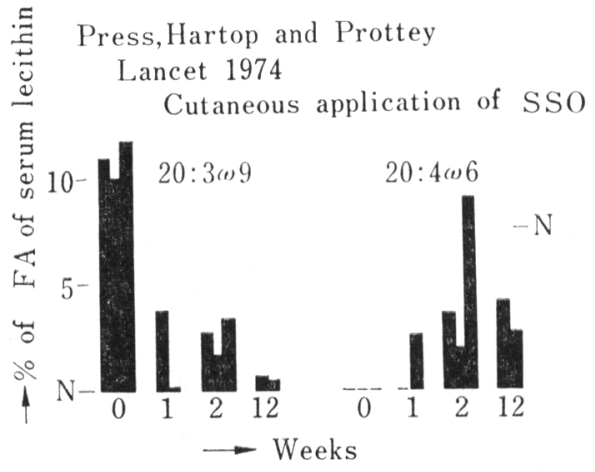

Fig.-12 The effects of dermal application of sunflower seed oil upon the 20:3 (1) 9 and 20: 4 (1) 6 conte. nts of serum lipids.

All these evidences show that EFA are necessary for human nutrition and metabolism, and it then is evident that adequate amounts of EFA must be provided by the diet to insure health. The minimum requirement is in the order of $1 \%$ of calories of linoleic acid, but some individuals may require considerably more. The requirement for optimum nutrition has not been defined, but our opinion it would be wise to consume that 5 to $10 \%$ of calories of linoleate. High levels of polyunsaturated acids have some adverse effects, probably related to the exhaustion of limited 
tocopherol. Very high levels of PUFA in rats cause dermal symptoms to appear and growth to diminish. Hence, care should be exercised not to consume too high proportions of PUFA. If a little is good, it does not necessarily follow that much is better.

The question is often asked how much and what kind of fat should be included in the diet to assure an adequate intake of EFA. The American population consumes approximately $40 \%$ of its calories as fat. If all of this fat were consumed as butter fat, the intake of linoleic acid would be 0.5 to $1 \%$ of calories. This is shown superimposed on the familiar triene/tetraene curve in Fig.-13. If, on the other hand, all the dietary fat were sunflower seed oil, corn oil or cottonseed oil, about $20 \%$ of calories of linoleic acid would be provided. Other sources of dietary fat range in between these limits. No population, no culture, and even no individual consumes its fat all from one source, so it is unlikely that a mixed diet could result in an EFA deficiency in humans. Only in those cultures which depend heavily on cow's milk fat and body fats of ruminant animals as a major source of calories, is it likely that marginal EFA deficiency could arise. Some individuals may have greater requirements than others because of inherited errors in metabolism, and such cases should be investigated to learn whether high intakes of EFA would help correct their deficiencies. For the great majority of the rest of us, whether we are Occiden-

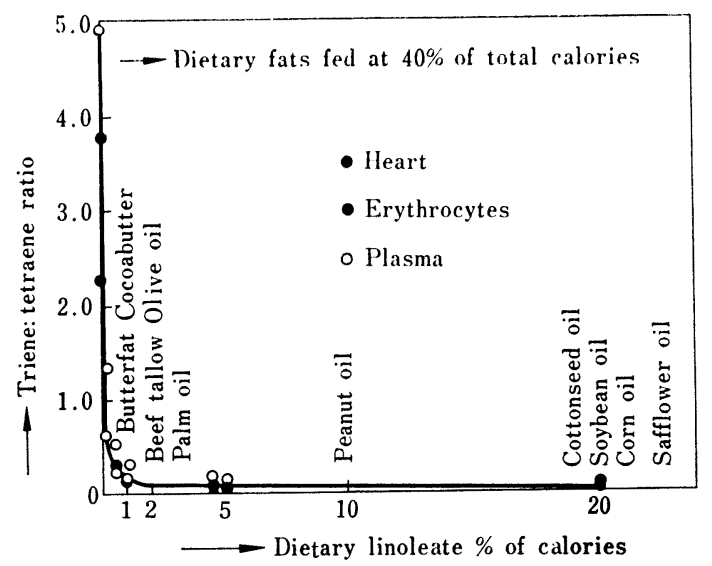

Fig.-13 Efficacy of various dietary fats in providing essential fatty acid when fed at $40 \%$ of total calories. tal or Oriental, the danger of EFA deficiency is minimal. We need only extend the principles of balanced nutrition to include a balanced variety of dietary fats in the human diet.

The reader is referred to recent reviews on the subjects of essential fatty acids in animals ${ }^{16), 17)}$ and humans ${ }^{18}$ for additional background information.

\section{Acknow ledgment}

Research from The Hormel Institute reviewed in this report was supported by grants from the National Institutes of Health (AM 07524), the National Dairy Council, and The Hormel Foundation.

(Recieved on Dec. 16, (1974))

\section{References}

1) G.O. Burr and M.M. Burr, J. Biol. Chem., 82, 345 (1929)

2) W.R. Brown, A.E. Hansen, G.O. Burr and I.J. McQuarrie, Nutr., 16, 511 (1938)

3) A.E. Hansen, E.M. Knott, H.F. Wiese, E. Shaperman and I. McQuarrie, Amer. J. Diseases Children, 73, 1 (1947)

4) A.E. Hansen, R.A. Stewart, G. Hughes and L. Söderhjelm, Acta Paediat. Suppl., 137, 51, 1 (1962)

5) R.T. Holman, J. Nutr., 70, 405 (1960)

6) A.E. Hansen, H.F. Wiese, A.N. Boelsche, M.E. Haggard, D.J.D. Adam and H. Davis, Pediatrics, 31 (Suppl. 1, Part 2), 171 (1963)

7) R.T. Holman, W.O. Caster and H.F. Wiese, Amer. J. Clin. Nutr., 14, 70 (1964)

8) R.T. Holman, W.O. Caster and H.F. Wiese, Amer. J. Clin. Nutr., 14, 193 (1964)

9) C. Pudelkewicz, J. Seufert and R.T. Holman, $J$. Nutr., 94, 138 (1968)

10) R.T. Holman, H.W. Hayes, A. Rinne and L. Söderhjelm, Acta Paed. Scand., 54, 573 (1965)

11) R. Cash and C.K. Berger, .J. Pediat., 74, 717 (1969)

12) J.R. Paulsrud, L. Pensler, C.F. Whitten, S. Stewart and R.T. Holman, Amer. J. Clin. Nutr., 25, 897 (1972)

13) F.D. Collins, A.J. Sinclair, J.P. Royle, D.A. Coats, A.T. Maynard and R.F. Leonard, Nutr. Metab., 13, 150 (1971)

14) M.D. Caldwell, H.T. Jonnson and H.B. Othersen, Pediatrics, 81, 894 (1972)

15) M. Press, P.J. Hartop and C. Prottey, Lancet, 1, 594 (1974)

16) R.T. Holman, "Prog. Chem. Fats Other Lipids", Vol. 9, p. 279-348 (1971), Pergamon Press, Oxford

17) “Prog. Chem. Fats Other Lipids", Vol. 9, p. 611682 (1971), Pergamon Press, Oxford

18) L. Söderhjelm, H.F. Wiese and R.T. Holman, "Prog. Chem. Fats Other Lipids", Vol. 9, p. 557585 (1971), Pergamon Press Oxford 